\title{
Ankle-Brachial Index
}

National Cancer Institute

\section{Source}

National Cancer Institute. Ankle-Brachial Index. NCI Thesaurus. Code C87304.

The ratio of ankle systolic pressure to brachial systolic pressure, used to assess arterial insufficiency in the lower extremities. 